Ocean Sci. Discuss., doi:10.5194/os-2016-1, 2016

Manuscript under review for journal Ocean Sci.

Published: 12 February 2016

(c) Author(s) 2016. CC-BY 3.0 License.

\title{
A harmonic projection and least-squares method for quantifying Kelvin wave activity
}

\author{
Andrew Delman ${ }^{1}$, Janet Sprintall ${ }^{1}$, Julie McClean ${ }^{1}$, and Lynne Talley ${ }^{1}$ \\ ${ }^{1}$ Scripps Institution of Oceanography, University of California-San Diego, La Jolla, California, \\ USA. \\ Correspondence to: Corresponding author: Andrew S. Delman, Scripps Institution of \\ Oceanography, University of California-San Diego, La Jolla, California, USA. \\ (adelman@ucsd.edu)
}

\begin{abstract}
A new method for isolating the equatorial and coastal Kelvin wave signal from alongtrack satellite altimetry data is presented and applied to sea level anomaly (SLA) observations in the tropical Indian Ocean. The method consists of sequential projections onto the SLA data, starting with meridional or cross-shore Kelvin wave profiles derived from shallow water theory ( $y$-projections).

5 Next, Fourier basis functions in $x-t$ (along-waveguide distance and time respectively) space with the phase speed ranges of Kelvin and Rossby waves are projected onto the $y$-projections. After projections in all three dimensions have been carried out, least-squares methods are applied to optimize the non-orthogonal basis function coefficients and minimize the misfit of their along-waveguide forcing and dissipation. Lastly, the westward-propagating (Rossby wave-related) signals are removed, generating a Kelvin wave coefficient $K$ that represents Kelvin wave activity. Along the Indian Ocean equatorial-coastal waveguide, Hovmöller diagrams of $K$ show reduced high-wavenumber noise compared to analogous diagrams of pre-processed sea level anomaly. Results from a Monte Carlo simulation demonstrate that Kelvin wave signals generated a priori can be effectively isolated even when superimposed with strong Rossby waves; the signs of all but the weakest Kelvin waves are diagnosed correctly in over $90 \%$ of cases. When the method is applied to 21 years of satellite observations and the SLA signal associated with $K$ is removed, the large residual in the equatorial SLA signal has a spatial distribution consistent with wind-forced Rossby waves. The equatorial SLA variability in the western part of the basin is poorly correlated with the SLA field associated with $K$, as the superimposed SLA profile of Rossby waves can distort the true origin locations of Kelvin waves in the raw SLA field. Therefore, this method offers improved tracking of Kelvin waves compared to the raw SLA dataset, and may provide the opportunity to study weakly nonlinear aspects of these waves by comparison with linear models.
\end{abstract}


Ocean Sci. Discuss., doi:10.5194/os-2016-1, 2016

Manuscript under review for journal Ocean Sci.

Published: 12 February 2016

(c) Author(s) 2016. CC-BY 3.0 License.

\section{Introduction}

The quantification of ocean variability associated with equatorial long waves is a topic of great importance for understanding the tropical ocean and its role in climate. Since the advent of satellite altimetry, the surface manifestations of these waves and the wind forcing driving them have been tracked in datasets that now comprise over 20 years of continuous global coverage (e.g., Delcroix et al., 1994; Susanto et al., 1998; Boulanger and Menkes, 1999; Drushka et al., 2010). However, to use these observations to better understand the behavior of these planetary waves and their relationship to climate variability, analysis techniques are needed that target the specific signatures of Kelvin and Rossby waves in satellite observations. In particular, the present study was motivated by a need to quantify the relative presence of upwelling vs. downwelling Kelvin waves in the equatorial Indian Ocean and along the coasts of Sumatra and Java, where they are influential in the evolution of Indian Ocean Dipole events (Delman et al., submitted).

A variety of techniques have been employed to quantify equatorial long wave activity from satellite observations; these range from the application of sophisticated data assimilation techniques to meridional projections of sea level anomaly (SLA) data. The data assimilation approaches generally use a linear wave-propagation model, along with Kalman filters (e.g., Miller and Cane, 1989; Fu et al., 1993) or adjoints (e.g., Thacker and Long, 1988; Long and Thacker, 1989a, b) to incorporate observations. These techniques are particularly useful for cases where observations are sparse and error-prone, as is often the case for in-situ measurements, and also during the earlier years of satellite observations when spatial resolution was low (e.g., Geosat). As the spatial and temporal coverage of altimeter-derived remote sensing data increased, it was conceivable to estimate Kelvin and Rossby wave activity using solely meridional projections of SLA data, or a combination of SLA and current observations. Cane and Sarachik (1981) showed that vectors containing SLA and surface current profiles associated with a given vertical Kelvin wave mode and its associated meridional Rossby wave modes are orthogonal; this orthogonality provided the basis for an equatorial wave decomposition in numerous studies (e.g., Delcroix et al., 1994; Yuan et al., 2004; Yuan and Liu, 2009). Boulanger and Menkes (1995, 1999), BM9599 hereafter, also carried out a decomposition using only meridional projections of SLA data that were reasonably consistent with projections derived from in-situ moorings. However, the decomposition of Kelvin and Rossby wave modes based on meridional projections of SLA alone are not orthogonal, and as Yuan et al. (2004) notes, this necessitates the inversion of an ill-conditioned matrix. An alternative approach used complex EOFs of SLA to separate Rossby and Kelvin wave signals in the equatorial Pacific (Susanto et al., 1998); one limitation of this method is that complex EOFs by definition constrain the along-waveguide and across-waveguide length scales of the waves, while shallow-water theory only constrains the across-waveguide length scale.

Here we build on the methodology of BM9599, by using the approximate phase speeds as well as cross-waveguide profiles to isolate the Kelvin wave signal. Starting with the SLA meridional 
Ocean Sci. Discuss., doi:10.5194/os-2016-1, 2016

Manuscript under review for journal Ocean Sci.

Published: 12 February 2016

(c) Author(s) 2016. CC-BY 3.0 License.

60 projections of BM9599, we apply harmonic projections in the along-waveguide direction and in time, followed by a least-squares fit to optimize the non-orthogonal projection coefficients. The result is a Kelvin wave coefficient $K$ that approximates Kelvin wave generation and dissipation along the waveguide, and can be used to track coastal as well as equatorial Kelvin waves. The method as described is focused only on an accurate representation of Kelvin (not Rossby) wave activity, though an extension of these techniques might enable a comprehensive decomposition of equatorial waves (as discussed in Section 4). The paper is structured as follows: Section 2 describes the satellite data used, and the harmonic projection and least-squares method that results in the Kelvin wave coefficient $K$. Section 3 estimates errors associated with the computation of $K$ using a Monte Carlo simulation, and discusses qualitative and quantitative analyses of satellite observations to assess how effectively $K$ describes Kelvin wave activity along the Indian Ocean waveguide. Section 4 summarizes the strengths and weaknesses of the method, and considers the possibility of extending the method to quantify Rossby wave activity.

\section{Method}

\subsection{Data}

75 Our methodology quantifies Kelvin wave activity using AVISO Ssalto/Duacs alongtrack SLA data, specifically those from the TOPEX/Poseidon, Jason-1, and Jason-2 satellites. These satellites repeat their orbit over a given track approximately every 10 days, and the data have near-continuous coverage from September 1992 to December 2013. The reason for using alongtrack as opposed to the frequently-used gridded product is the increased spatial resolution in the along-track direction, $\sim 1 / 10^{\circ}$ compared to $1 / 3^{\circ}$ for gridded data. One of the advantages of this method is its utility for tracking waves in their transition from equatorial to coastal Kelvin waves. However, quantifying coastal Kelvin waves requires higher spatial resolution, as the baroclinic radius of deformation shrinks from $\sim 400 \mathrm{~km}$ at the equator to $\sim 100 \mathrm{~km}$ at $10^{\circ} \mathrm{S}$. The disadvantage of using the alongtrack data is the large spacing between tracks in the zonal/alongshore direction $(\sim 300 \mathrm{~km}$ along the coast), but the spacing is still small relative to the along-waveguide length scale of Kelvin waves near the equator, typically $>1000 \mathrm{~km}$.

Due to the anisotropy of equatorial-coastal long waves, the offset angle between satellite tracks and meridional cross-sections at the equator is likewise considered to be negligible, and both ascending and descending tracks are used in the analysis. Along the Sumatra and Java coasts, only ascending (SW-NE oriented) tracks are used in Kelvin/Rossby wave projections to best approximate a cross-shore profile. For computational expediency in the least-squares part of the solution, the method was applied to overlapping two-year subsets of the full data record, with each subset overlapping with the next one by a year. The results from each subset were then patched together using a tapered weighted averaging in the overlapping year to create a continuous field of $K$ values 
Ocean Sci. Discuss., doi:10.5194/os-2016-1, 2016

Manuscript under review for journal Ocean Sci.

Published: 12 February 2016

(c) Author(s) 2016. CC-BY 3.0 License.

for the 21-year period of record (i.e., with 20 subsets patched together). For comparison purposes and to present clear visual snapshots of variability in the Indian Ocean basin, gridded maps of SLA (MSLA) (Ducet et al., 2000) were also used to generate some of the figures in this paper.

\subsection{Kelvin wave $y$-projections}

The first step in the computation of the Kelvin wave coefficient $K$ is to calculate the projection of the SLA data onto a meridional or cross-shore profile of a baroclinic Kelvin wave based on linear shallow-water theory (e.g., Gill and Clarke, 1974; McCreary, 1981). We refer to this as the $y$-projection; for an equatorial Kelvin wave it is the same Gaussian profile given in Appendix A2 of Boulanger and Menkes (1995), but our analysis also considers coastal Kelvin waves for which the wave profile transitions to a decaying exponential away from the equator. For an equatorial-coastal Kelvin wave the profile is

$h_{K}(y)=h_{0} \exp \left[-\frac{\beta \cos \phi}{2 c} y^{2} \pm \frac{f_{0}}{c} y\right]$

where $y$ is the perpendicular distance relative to the equator or the coastline, $h_{0}$ is the amplitude (i.e., peak value) of the wave, $f_{0}$ is the Coriolis parameter at the latitude where the profile intersects the coast, and $\phi$ is the angle of orientation of the coast relative to the east-west axis $\left(f_{0}=0\right.$ and $\phi=0$ for equatorial Kelvin waves). The sign in front of $\left(f_{0} / c\right) y$ for coastal Kelvin waves is chosen such that the term is always negative. As our focus here is on Indian Ocean Kelvin waves that are deflected to the south of the equator, $y$ is negative and decreasing away from the coast, and $f_{0}<0$, so the sign is negative. The value of $c$ for the meridional/cross-shore profiles in this analysis was taken to be $2.5 \mathrm{~m} \mathrm{~s}^{-1}$. This value of $c$ lies between the first-and second-mode baroclinic phase speeds for Kelvin waves in the region, as these two modes account for most Kelvin waves observed in Indian Ocean SLA (Drushka et al., 2010). However, using $c=2.0 \mathrm{~m} \mathrm{~s}^{-1}$ or $3.0 \mathrm{~m} \mathrm{~s}^{-1}$ does not produce a substantially different result.

Applied to the altimetry data, the Kelvin wave $y$-projection is given by

$K_{y}=\frac{1}{2} \int_{-r}^{r}\left(h_{\mathrm{SLA}}-\overline{h_{\mathrm{SLA}}}\right) \frac{h_{K}-\overline{h_{K}}}{h_{0}} d y$

for equatorial Kelvin waves and

$K_{y}=\int_{-r}^{0}\left(h_{\mathrm{SLA}}-\overline{h_{\mathrm{SLA}}}\right) \frac{h_{K}-\overline{h_{K}}}{h_{0}} d y$

for coastal Kelvin waves south of the equator, where $h_{\mathrm{SLA}}$ is the alongtrack altimetry profile, and $r$ is the radius for the profile projection. The overbar indicates the meridional $\bar{a}=1 /(2 r) \int_{-r}^{r} a d y$ or 
Ocean Sci. Discuss., doi:10.5194/os-2016-1, 2016

Manuscript under review for journal Ocean Sci.

Published: 12 February 2016

(c) Author(s) 2016. CC-BY 3.0 License.

cross-shore $\bar{a}=1 /(r) \int_{-r}^{0} a d y$ mean (for equatorial and coastal waves respectively) of the profile $a$ over the range being integrated. For the $r$ value, we used $5^{\circ}$ of latitude for equatorial Kelvin waves; $r$ was then tapered to a distance equivalent to $3^{\circ}$ of latitude along the coasts of Java and Nusa Tenggara to account for the smaller radius of deformation. We note that $K_{y}$ is an integrated measure of the sea level displacement; this type of measure is a more consistent indicator of Kelvin wave activity in the equatorial-coastal transition than peak amplitude, since without substantial dissipation, the peak amplitude of the wave tends to increase poleward as the radius of deformation decreases (Figure 1).

\subsection{Projection using harmonic basis functions in $x$ and $t$}

After the Kelvin wave $y$-projections $K_{y}$ are computed, the next step in our approach is to project $K_{y}$ onto two-dimensional Fourier basis functions in along-waveguide distance $x$ and time $t$. One method of separating these components is to assume that a vector $\mathbf{b}$ consisting of the alongtrack Kelvin wave projections $K_{y}$ can be explained as a linear combination of two-dimensional Fourier basis functions

$\mathbf{A m}=\mathbf{b}$

where the columns of $\mathbf{A}$ are the basis functions $A_{m, n}^{\cos }=\cos \left[2 \pi\left(k_{m} x-f_{n} t\right)\right]$ and $A_{m, n}^{\sin }=\sin \left[2 \pi\left(k_{m} x-f_{n} t\right)\right]$

and $x$ and $t$ are along-waveguide distance and time respectively; the Fourier coefficients to be solved for are contained in the vector $\mathbf{m}$.

Basis functions $A_{m, n}$ that propagate from one side of the basin to the other at constant amplitude are most effective at representing Kelvin waves that similarly propagate across the basin with little change in amplitude. Kelvin waves that are forced and dissipate within the domain, especially with the low wavenumbers common to Kelvin waves, may have some of their energy aliased into westward-propagating signals. To resolve this aliasing issue, we introduce an additional tapering parameter $s$ to the basis functions (Figure 2). The basis functions $A_{m, n, s}$ take the form

$A_{m, n, s}= \begin{cases}0, & x \leq x_{s}-\Delta x \\ \left(1-\frac{x_{s}-x}{\Delta x}\right) A_{m, n}, & x_{s}-\Delta x<x<x_{s} \\ A_{m, n}, & x \geq x_{s}\end{cases}$

The tapering location $x_{s}$ is varied at intervals of $\Delta x=600 \mathrm{~km}$ throughout the span of the waveguide, corresponding to the shortest wavelengths resolved along the coastal part of the waveguide (along the equator the effective Nyquist wavenumber is higher with more satellite tracks used). For $s=1$, $x_{s}=x_{W}$ the western boundary, while for $s>1, x_{s}=x_{W}+(s-1) \Delta x$. The forcing and dissipation of a wave within the domain can be expressed as the superposition of basis functions with varying $s$-values. 
Ocean Sci. Discuss., doi:10.5194/os-2016-1, 2016

Manuscript under review for journal Ocean Sci.

Published: 12 February 2016

(c) Author(s) 2016. CC-BY 3.0 License.

Furthermore, to reduce the number of basis functions and make the subsequent least-squares with the waves we expect to observe using satellite altimetry. Therefore only basis functions $A_{m, n, s}$ corresponding to phase speeds $c_{m, n}=f_{n} / k_{m}$ typical of Kelvin waves $\left(1.5 \mathrm{~m} \mathrm{~s}^{-1} \leq c_{m, n} \leq 3.5 \mathrm{~m}\right.$ $\left.\mathrm{s}^{-1}\right)$, Rossby waves $\left(-1.2 \mathrm{~m} \mathrm{~s}^{-1} \leq c_{m, n} \leq-0.4 \mathrm{~m} \mathrm{~s}^{-1}\right)$, and stationary signals $\left(k_{m}=0\right.$ or $\left.f_{n}=0\right)$ are included in $A_{m, n, s}$, while the other basis functions are excluded. This phase-speed limitation reduces the number of basis functions to approximately twice the number of $K_{y}$ values in $\mathbf{b}$. The tapered basis functions in $A_{m, n, s}$ corresponding to the phase speed ranges are projected onto the vector $\mathbf{b}$ containing the $K_{y}$ values. First, a least-squares planar fit to $\mathbf{b}$ is removed, and then the basis functions are projected onto $b$

$\mathbf{m}^{\mathbf{P}}=\mathbf{A}^{T} \mathbf{b}$

producing a vector $\mathbf{m}^{\mathbf{P}}$ of data projections that can be used to solve for the basis function coefficients

$\mathbf{m}$ of identical size. For the projection values to be unbiased with respect to $s$, the projections are normalized by the size of the nonzero domain, expressed as a normalizing vector $\mathbf{n}^{\mathbf{P}}$, with $n_{m, n, s}^{P}=$ $(2 / N) x_{\max }\left[x_{\max }-\left(x_{s}-0.5 \Delta x\right)\right]^{-1}$, and $N=\operatorname{length}(\mathbf{b})$. Accordingly, the normalized vector of basis function projections is

$\mathbf{m}^{\mathbf{n P}}=\mathbf{n}^{\mathbf{P}} \mathbf{m}^{\mathbf{P}}=\mathbf{n}^{\mathbf{P}} \mathbf{A}^{T} \mathbf{b}$

\subsection{Least-squares optimization and removal of westward-propagating signals}

After the $x-t$ projections have been carried out and normalized, basis function coefficients are recovered from the data projections in $\mathbf{m}^{\mathbf{n P}}$ using least-squares methods; the solution is optimized to prevent a poorly-scaled solution with the cancellation of large coefficients in $\mathbf{m}$. In addition to constraining the size of the basis function coefficients $\mathbf{m}^{T} \mathbf{m}$, we chose to minimize the misfit of the rate of change in data projection values along the waveguide, $\partial \mathbf{m}^{\mathbf{n P}} / \partial s$, in order to constrain high-wavenumber variability within the domain. We also minimize the misfit of the untapered data projection values, $\left.\mathbf{m}^{\mathbf{n P}}\right|_{s=1}$, to the $s=1$ basis function coefficients. Hence the vector that we minimize the misfit for is

$\mathbf{w}^{T} \mathbf{D m}^{\mathbf{n P}}=\mathbf{w}^{T}\left[\begin{array}{c}\left.\mathbf{m}^{\mathbf{n P}}\right|_{s=1} \\ \left.\frac{\partial \mathbf{m}^{\mathbf{n P}}}{\partial s}\right|_{s>1}\end{array}\right]$

where $\mathbf{D}$ is the identity operator for $s=1$ projections, and the finite difference operator for $s>1$ projections. The column vector $\mathrm{w}$ can be used to weight the elements of $\mathbf{D m}^{\mathrm{nP}}$ relative to one another. In this case setting $\mathbf{w}$ to all ones was found to be sufficient, though accuracy may be gained 
Ocean Sci. Discuss., doi:10.5194/os-2016-1, 2016

Manuscript under review for journal Ocean Sci.

Published: 12 February 2016

(c) Author(s) 2016. CC-BY 3.0 License.

in certain areas by adjusting the weighting vector; we speculate about one such case in Section 4 . With $\mathbf{w}_{n}=1$ for all $n$, we miminize the misfit of $\mathbf{D m}^{\mathbf{n P}}$ and the size of $\mathbf{m}^{T} \mathbf{m}$ using the cost function

$L=\left[\mathbf{D n}^{\mathbf{P}} \mathbf{A}^{T}(\mathbf{A m})-\mathbf{D m}^{\mathbf{n P}}\right]^{T}\left[\mathbf{D n}^{\mathbf{P}} \mathbf{A}^{T}(\mathbf{A m})-\mathbf{D m}^{\mathbf{n P}}\right]+\lambda \mathbf{m}^{T} \mathbf{m}$

Setting $\lambda=0.1$ was found heuristically to produce the most credible reconstructions of the $K_{y}$ values in $\mathbf{b}$, while reducing noise at the highest wavenumbers. The coefficient vector $\mathbf{m}$ is then given by

$\mathbf{m}=\left[\left(\mathbf{D n}^{\mathbf{P}} \mathbf{A}^{T} \mathbf{A}\right)^{T} \mathbf{D n}^{\mathbf{P}} \mathbf{A}^{T} \mathbf{A}+\lambda \mathbf{I}\right]^{-1} \mathbf{D n}^{\mathbf{P}} \mathbf{A}^{T} \mathbf{A D m}^{\mathbf{n P}}$

Finally, all coefficients of $\mathbf{m}$ that correspond to westward-propagating basis functions, i.e., $\operatorname{sgn}\left(f_{n}\right)=$ $-\operatorname{sgn}\left(k_{m}\right)$, are set to zero. The resulting vector $\mathbf{m}_{\mathbf{K}}$ is used to reconstruct the $K_{y}$ field with the westward-propagating signals removed

$\mathbf{b}_{\mathbf{K}}=\mathbf{A} \mathbf{m}_{\mathbf{K}}$

where vector $\mathbf{b}_{\mathbf{K}}$ consists of the Kelvin wave coefficients $K$ as a function of $x$ and $t$. These computations are carried out for overlapping two-year subsets of the data, which are then merged to create a continuous field of $K$ values for the period Sept. 1992-Dec. 2013 covered by the alongtrack SLA dataset.

\section{Representations of Kelvin wave activity and error/variance estimates}

\subsection{Comparison of $K$ values with raw SLA}

To demonstrate how well $K$ represents Kelvin wave activity, we present a case study where we compare the raw SLA along the IO equatorial-coastal waveguide during the year 1997 to the $K_{y}$ and $K$ values for the same period (Figure 3). The $K_{y}$ and $K$ values are calculated from the alongtrack SLA data at points where the satellite tracks cross the waveguide, hence these values are presented as points in Fig. 3b-c, whereas the raw SLA data from the gridded product are contoured in Fig. 3a. During the May-July period, the predominant feature in the raw SLA (Fig. 3a) is an eastwardpropagating patch of elevated positive SLA, indicative of a downwelling Kelvin wave. However, the Kelvin wave $y$-projection (Fig. 3b) shows that this downwelling wave was both preceded and followed by upwelling Kelvin waves, both of which are much more evident in the Kelvin wave projection (Fig. 3b) than in the raw SLA (Fig. 3a). The $y$-projection still contains a number of westward-propagating signals (e.g., Jan.-Feb., and Oct.-Dec.) unrelated to Kelvin waves, and most likely represent Rossby waves flanking the equator. These westward-propagating signals are no 
Ocean Sci. Discuss., doi:10.5194/os-2016-1, 2016

Manuscript under review for journal Ocean Sci.

Published: 12 February 2016

(c) Author(s) 2016. CC-BY 3.0 License.

longer visible in the $K$ values for 1997 (Fig. 3c), and the trajectories of alternating upwelling and downwelling Kelvin waves are much more readily apparent.

The values of $K$ can also be re-projected back into two spatial dimensions, to reconstruct the component of the SLA field that is associated with Kelvin wave activity. The reconstructed $h_{K}$ is obtained by obtaining the wave amplitude $h_{0}$ associated with $K$

$h_{0}(x, t)=\frac{K(x, t)}{\int_{-r}^{0} \exp \left[-\frac{\beta \cos \phi}{2 c} y^{2}-\frac{f_{0}}{c} y\right] d y}$

and substituting into (1). A comparison of the reconstructed $h_{K}$ with gridded maps of SLA over a two-month period in 1997 (Figure 4) confirms that the Kelvin wave reconstruction is broadly consistent with the Kelvin wave activity suggested by the gridded SLA field, but also highlights some key differences. In late May and early June, an elevated SLA field persists in the eastern equatorial Indian Ocean (Fig. 4b-c), while the reconstructed $h_{K}$ indicates that the Kelvin wave activity is changing sign from positive to negative there (Fig. $4 \mathrm{~g}-\mathrm{h}$ ). In late June and early July, reconstructed $h_{K}$ indicates that upwelling Kelvin wave activity is being generated from approximately $60^{\circ} \mathrm{E}$ eastward (Fig. 4i-j), while SLA is only substantially depressed east of $90^{\circ} \mathrm{E}$ (Fig. 4d-e). The discrepancy during this latter period is likely accounted for by the influence of downwelling Rossby waves on the SLA field in the central Indian Ocean; these waves have positive SLA maxima near the north and south radii of deformation, and would also elevate SLA (to a lesser extent) at the equator. Therefore, this implies that in the raw SLA field in early July 1997 (Fig. 4e) the upwelling Kelvin wave still present in the central equatorial Indian Ocean would not be apparent; this has potential implications for understanding the timing of the upwelling wave and where it was forced.

\subsection{Monte Carlo-based error estimates}

In order to place uncertainty bounds on the method's capacity to remove westward-propagating wave activity from the Kelvin wave estimate, we carried out a Monte Carlo simulation. In this way the method could be applied to propagating waves whose amplitudes and $K$ values were known $a$ priori.

We created 100 years of randomly-generated basis function coefficients $\mathbf{m}$, using Cholesky factorization (e.g., Gentle, 1998) to construct $\mathbf{m}$ fields whose local covariance statistics in wavenumberfrequency $(k-f)$ space resemble values computed from the altimetry data, so that realistic Kelvin and Rossby wave signals could be generated. The $\mathbf{m}$ coefficients were adjusted so that their values are partially dependent on the local wave amplitude at the same wavenumber and frequency

$\left.\mathbf{m}\right|_{k, f, s}=\left[\left.C_{s} \sum_{s^{\prime}=1}^{s-1} \mathbf{m}\right|_{k, f, s^{\prime}}\right]+\mathbf{r}$

with $C_{s}$ the location-dependent adjustment parameter and $\mathbf{r}$ the Cholesky decomposition-based random component. The variances of the basis functions were also adjusted so that the distributions 
Ocean Sci. Discuss., doi:10.5194/os-2016-1, 2016

Manuscript under review for journal Ocean Sci.

Published: 12 February 2016

(c) Author(s) 2016. CC-BY 3.0 License.

of total Kelvin and Rossby wave variance along the waveguide are consistent with the variances obtained from satellite altimetry. Finally, after the artificially-generated eastward- and westwardpropagating signals were combined, a small amount of white noise was added to the $K_{y}$ fields; the variance of this noise is location-dependent and based on the variance in altimetry observations that could not be explained by either Kelvin or Rossby wave signals.

Once the artificial wave field was constructed, the harmonic projection and least-squares method described in sections 2.3-2.4 was applied to the artificial $K_{y}$ field, and the $K$ values derived from the basis function coefficients known a priori and deduced from the method were compared. An example of this for a given simulated year is shown in Figure 5; the artificially-generated $K_{y}$ field contains signals propagating in both directions, though for most of the year the westward-propagating Rossby waves appear to predominate (Fig. 5a). However, a consideration of the Kelvin wave signal $K_{\text {a priori }}$ in isolation (Fig. 5b) reveals that in addition to the very strong downwelling wave early in the year, a series of weak and moderate Kelvin waves propagate throughout the year. Many of these weaker waves are unidentifiable in the $K_{y}$ field with the Rossby wave signals superimposed (Fig. 5a). However, the reconstructed Kelvin wave signal $K_{\text {reconst }}$, computed by applying the harmonic projection and leastsquares method, recovers most of the weaker Kelvin waves in the $K_{\text {a priori }}$ signal and reproduces their approximate timing and intensity (Fig. 5c). In the few locations where visible discrepancies between $K_{\text {a priori }}$ and $K_{\text {reconst }}$ are present (e.g., the intensities of the Kelvin waves in March-April, east of $90^{\circ} \mathrm{E}$ ), high amplitude westward-propagating signals and/or sharp noisy gradients are present in the $K_{y}$ field.

We now consider the error that is present in the reconstructed signal $K_{\text {reconst }}$ relative to the original signal $K_{\text {a priori }}$, specifically $\epsilon=K_{\text {reconst }}-K_{\text {a priori }}$. When the $100-$ year artificial timeseries is analyzed, it is found that the normalized root-mean-square (RMS) error $\left\langle\epsilon^{2}\right\rangle^{1 / 2} /\left\langle K_{\text {reconst }}^{2}\right\rangle^{1 / 2}$ is dependent on location along the waveguide as well as whether the fields are spatially- and temporally-filtered (Figure 6a). (Here the angle brackets \langle\rangle denote temporal averaging over the entire 100-year time span of the simulation, but no spatial averaging other than filters applied prior to the error calculation.) The error in recovering the original Kelvin wave signal is highest near the equatorial-coastal transition of the waves, and on the eastern end of the domain; elsewhere it is confined to a fairly narrow range. However, the error magnitude also depends on whether a spatial or temporal averaging filter is applied prior to the error calculations. Except for the most error-prone regions, the error associated with unfiltered pointwise values of $K$ is $50 \%$ to $60 \%$ of the total standard deviation of $K$. If the $K$ has a spatial moving average (boxcar) filter applied, but temporal averaging is limited to 10 day ranges (the resolution of the original points), the normalized error decreases slightly in most

locations and is smoother across the waveguide. The error associated with 30-day moving averages of $K$ (a typical timescale for intraseasonal Kelvin waves) decreases more substantially, to 35\%-45\% in most locations. 
Ocean Sci. Discuss., doi:10.5194/os-2016-1, 2016

Manuscript under review for journal Ocean Sci.

Published: 12 February 2016

(c) Author(s) 2016. CC-BY 3.0 License.

The probability and cumulative distribution functions associated with errors in $K$ illustrate that errors of the same magnitude as the Kelvin waves themeselves are infrequent when a 30-day moving average filter is applied (Fig. 6b). Relative to the total standard deviation in filtered $K, \sigma_{K}$, the magnitude of the errors only exceed $0.5 \sigma_{K}$ about $10 \%$ of the time (either positive or negative), and only exceed $1 \sigma_{K}$ about $2.5 \%$ of the time. In this simulation, $\sigma_{K} \approx 1.9 \times 10^{4} \mathrm{~m}^{2}$, so the error magnitude is less than $1 \times 10^{4} \mathrm{~m}^{2}$ over $90 \%$ of the time. If the error is considered relative to the magnitude of the filtered reconstructed $K_{\text {reconst }}$ at each location and time, the error variance is somewhat larger. Even so, with the weakest Kelvin waves $\left(\left|K_{\text {reconst }}\right|<0.3 \sigma_{K}\right)$ excluded, the error will only result in misdiagnosing the sign of most Kelvin waves (i.e., $\epsilon / K_{\text {reconst }}>1$ ) approximately $8.5 \%$ of the time. Moreover, the $8.5 \%$ incidence decreases further if the threshold for excluding weak Kelvin waves is raised (approximately $5.5 \%$ for a $0.5 \sigma_{K}$ threshold and $2 \%$ for a $1 \sigma_{K}$ threshold); thus sign misdiagnosis using this method is rarely a problem for moderate and strong Kelvin waves.

\subsection{Kelvin wave-related and unrelated SLA characteristics}

For a more comprehensive view of the variability encapsulated by the Kelvin wave coefficient $K$ described here, we also consider the reconstructed Kelvin wave-associated SLA field $h_{K}$, in the context of the total SLA field $h_{\mathrm{SLA}}$ observed by satellites over the 21-year period from 1992 to 2013. The SLA at each point in space and time can be considered as the sum of the reconstructed $h_{K}$ and a residual $h_{\text {res }}$

$h_{\mathrm{SLA}}=h_{K}+h_{\mathrm{res}}$

with $h_{\text {res }}$ in theory encompassing contributions to the SLA field from processes unrelated to Kelvin waves, including Rossby and other planetary waves. Figures 7a,b illustrates the variances of $h_{K}$ and $h_{\text {res }}$ respectively, normalized by $h_{\mathrm{SLA}}$. The variance ratios of both $h_{K}$ (Fig. 7a) and $h_{\text {res }}$ (Fig. 7b) to $h_{\mathrm{SLA}}$ exceed 1 along some parts of the waveguide, though this is more commonly the case with $h_{\text {res }}$. (NB: The variance ratios can exceed 1 , since the $h_{K}$ and $h_{\text {res }}$ fields are generally not orthogonal. Thus we do not describe the variance ratios as "explained variance" in the traditional sense, but rather compare the variances attributed to Kelvin waves vs. the residual to examine whether the residual signal is consistent with other phenomena such as Rossby waves.)

Additionally, we compute the correlation between $h_{K}$ and the total $h_{\mathrm{SLA}}$ and $h_{\text {res }}$ fields (Figure 8 ), to consider whether the sign of Kelvin wave activity covaries with that of the total SLA field and the residual. The effective degrees of freedom $N^{*}$ for the correlations in Fig. 8 were computed from the decorrelation timescales of $h_{K}$. With 21 years of data, values of $N^{*}$ range from approximately 50 to 500 over the spatial domain, with decorrelation timescales ranging from intraseasonal to semiannual. (For $N^{*}=50$, correlation coefficient magnitudes exceeding 0.23 exceed the $95 \%$ confidence threshold; for $N^{*}=500$ this threshold is approximately 0.08.) The correlation of $h_{K}$ to $h_{\mathrm{SLA}}$ along 
Ocean Sci. Discuss., doi:10.5194/os-2016-1, 2016

Manuscript under review for journal Ocean Sci.

Published: 12 February 2016

(c) Author(s) 2016. CC-BY 3.0 License.

the waveguide (Fig. 8a) is strongly positive in the eastern part of the basin and along the coast, but insignificant or negative in the western part of the basin. The correlation of $h_{K}$ to $h_{\text {res }}$ is negative over nearly the entire domain, suggesting the tendency of $h_{K}$ and $h_{\text {res }}$ to be of opposite sign and explaining how $h_{\text {res }}$ in particular can have a much larger variance than the total SLA field.

The variance ratios (Fig. 7) and correlations (Fig. 8) suggest different contributions from $h_{K}$ and $h_{\text {res }}$ variability in at least four distinct regions along the waveguide. In the western and central parts of the equatorial basin, even the maximum variances of $h_{K}$ near the equator are only slightly more than half the variance associated with $h_{\text {res }}$. In this equatorial region, it is likely that most of $h_{\text {res }}$ can be attributed to Rossby waves; indeed a linear wind-forced model of equatorial waves (Nagura and McPhaden, 2010) has shown that in the western part of the basin, Rossby waves are associated with a higher SLA standard deviation than Kelvin waves, even at the equator where Kelvin wave variability peaks. The correlation of $h_{K}$ and $h_{\text {res }}$ (Fig. 8) is also strongly negative here, consistent with the expected response of the ocean to a uniform zonal wind forcing, which would generate Kelvin and Rossby waves of opposite sign. In the eastern part of the basin and along the coast of Sumatra, the variances of $h_{K}$ and $h_{\text {res }}$ are more comparable, though this does not quite resemble the results from the linear forced model (Nagura and McPhaden, 2010) which show a much larger component of SLA due to Kelvin waves than Rossby waves. Near the coast of Java, the variance of $h_{K}$ is much larger than that of $h_{\text {res }}$, suggesting that most of the SLA variability in this area can be attributed to coastal Kelvin waves. Along Nusa Tenggara (NT) the variance of $h_{\text {res }}$ is once again comparable to or greater than $h_{K}$; this may be due in part to the complexity of the islands and bathymetry here. It is also likely that the computation of $K$ does not accurately resolve the splitting and diversion of Kelvin wave energy through Lombok Strait between Java and NT (e.g., Syamsudin et al., 2004; Drushka et al., 2010), since the least-squares fit exhibits a preference for slow tapering of $K$ rather than the abrupt change in wave activity associated with the narrow strait.

Finally, the lack of a robust correlation between $h_{K}$ and $h_{\mathrm{SLA}}$ along the equator in the western part of the basin (Fig. 8) implies that using raw SLA to track Kelvin wave propagation may not accurately represent where waves originate. Namely, SLA crests and troughs that only become clearly apparent in the eastern part of the basin may actually have origins further west; some specific cases of this are evident when comparing the SLA and $K$ values for 1997 (Fig. 3a, c).

\section{Conclusions}

The harmonic projection and least-squares method outlined here produces a measure of Kelvin wave activity that can be applied directly to satellite observations of SLA, not only along the equator, but also along low-latitude coastal waveguides. The method removes the westward-propagating signals (i.e., Rossby wave signals) along such waveguides, and produces $K$ coefficients that represent the time variability of Kelvin wave activity at each location along the waveguide. When filtered to re- 
Ocean Sci. Discuss., doi:10.5194/os-2016-1, 2016

Manuscript under review for journal Ocean Sci.

Published: 12 February 2016

(c) Author(s) 2016. CC-BY 3.0 License.

move sub-monthly temporal variability, the values of $K$ have an RMS error of approximately 0.4 times the local standard deviation of $K$ at most points along the waveguide; excluding the weakest waves, the method also diagnoses the sign of the Kelvin waves correctly over $90 \%$ of the time. A decomposition of the near-equatorial SLA into Kelvin wave-associated and residual components generates a residual field generally consistent with the activity of wind-forced Rossby waves, which tend to be of the opposite sign of the Kelvin waves on which they are superimposed. Therefore the use of this method helps to isolate the Kelvin wave-associated SLA signal; it also allows for some variation in the phase speed of the waves, so a comparison of $K$ values with the results of linear wind-forced models of equatorial waves (e.g., Yu and McPhaden, 1999; Nagura and McPhaden, 2010) may be useful in studying some weakly nonlinear aspects of Kelvin waves.

It is notable that the SLA along the equator in the western Indian Ocean is not robustly correlated with Kelvin wave activity deduced from this method, a result that has important implications for the interpretation of SLA variability at the equator. The use of this method helps show that the SLA signal of numerous intraseasonal Kelvin waves can be traced to the western equatorial Indian Ocean and co-located with zonal wind stress forcing (e.g., Delman et al., submitted); therefore the lack of readily identifiable eastward-propagating sea level anomalies at the equator at a given time does not necessarily imply that Kelvin waves are absent. Rossby waves may be obscuring the Kelvin waves' signal on the western side of the basin, and the computation of $K$ may assist in tracking Kelvin waves from their true generation region.

We also observe two limitations of the harmonic projection/least-squares method in the form presented here, and consider how these might be addressed. The first is the difficulty of resolving Kelvin wave activity to the east of Lombok Strait $\left(\sim 115^{\circ} \mathrm{E}\right)$, as evidenced by the abrupt increase in residual variance $h_{\text {res }}^{2}$ (Fig. 7b) and decrease in $h_{K}-h_{\text {SLA }}$ correlation (Fig. 8a) from the Java to the NT coastline. Prior analyses of altimetric SLA (Syamsudin et al., 2004; Drushka et al., 2010) indicate that about 30-50\% of the Kelvin wave energy from the Java coastline continues eastward along the NT coastline; the rest of the energy is presumed to transit north through Lombok Strait. In terms of our method, which does not track Kelvin wave energy through the strait, this would require an abrupt "dissipation" of Kelvin wave activity at Lombok Strait, which is likely not resolved by our tapered basis functions. Moreover, the overall skill of the method decreases approaching the eastern boundary of our domain and the complex topography of the Savu Sea region. One possible way to resolve the abrupt change in Kelvin wave energy across the strait using our method is to adjust the weighting $\mathbf{w}$ of the misfit of the vector in (8). Namely, the misfit for the tapers that span the Lombok Strait region could be weighted more heavily, so that there is less tendency for the model to continue steady propagation of Kelvin waves past the strait. Additionally, a finer tapering scale (e.g., $\Delta x=$ $300 \mathrm{~km}$ instead of $600 \mathrm{~km}$ ) could be adopted in this particular region, though errors due to altimetry interactions with land may still present challenges. 
Ocean Sci. Discuss., doi:10.5194/os-2016-1, 2016

Manuscript under review for journal Ocean Sci.

Published: 12 February 2016

(c) Author(s) 2016. CC-BY 3.0 License.

The second issue is that this method was developed with the specific goal of isolating Kelvin waves, while the SLA signal due to Rossby waves is treated as a residual. By contrast, linear windforced models and prior studies that have used SLA projections have sought to quantify the activity of Kelvin and gravest-mode equatorial Rossby waves simultaneously. The variance ratios (Fig. 7) and correlations (Fig. 8) suggest that the majority of the residual SLA field along the equator can be attributed to Rossby wave activity, and in principle there is no reason why our method could not be expanded to specifically target Rossby waves as well. One possible alteration to our method is to carry out the $y$-projections and the $x, t$-projections simultaneously; i.e., link each propagating basis function to the $y$-profile of a Kelvin or Rossby wave mode depending on its phase speed. Isolating the SLA displacement associated with each mode would allow for a more complete picture of equatorial dynamics to be deduced from satellite altimetry, and this altered method will be explored in a future study.

Acknowledgements. Andrew Delman (ASD) was supported by a NASA Earth and Space Science Fellowship, grant number NNX13AM93H. Janet Sprintall (JS) and Julie McClean (JLM) were supported by NASA award number NNX13AO38G. Lynne Talley (LDT), JLM, and ASD were also supported by NSF grant OCE0927650. The altimeter products were produced by Ssalto/Duacs and distributed by Aviso, with support from CNES (http://www.aviso.oceanobs.com/duacs/). Computations were carried out on the Geyser cluster within the Yellowstone computing environment hosted by the National Center for Atmospheric Research (NCAR). 
Ocean Sci. Discuss., doi:10.5194/os-2016-1, 2016

Manuscript under review for journal Ocean Sci.

Published: 12 February 2016

(c) Author(s) 2016. CC-BY 3.0 License.

\section{References}

Boulanger, J.-P. and Menkes, C.: Propagation and reflection of long equatorial waves in the Pacific Ocean during the 1992-1993 El Niño, J. Geophys. Res., 100, 25,041-25,059, 1995.

Boulanger, J.-P. and Menkes, C.: Long equatorial wave reflection in the Pacific Ocean from TOPEX/POSEIDON data during the 1992-1998 period, Clim. Dyn., 15, 205-225, 1999.

Cane, M. and Sarachik, E.: The response of a linear baroclinic equatorial ocean to periodic forcing, J. Mar. Res., 39, 651-693, 1981.

Delcroix, T., Boulanger, J.-P., Masia, F., and Menkes, C.: Geosat-derived sea level and surface current anomalies in the equatorial Pacific during the 1986-1989 El Niño and La Niña, J. Geophys. Res., 99, 25,09325,107, 1994.

400 Delman, A. S., Sprintall, J., McClean, J. L., and Talley, L. D.: Anomalous Java cooling at the initiation of positive Indian Ocean Dipole events, J. Geophys. Res. Oceans, submitted.

Drushka, K., Sprintall, J., Gille, S. T., and Brodjonegoro, I.: Vertical structure of Kelvin waves in the Indonesian Throughflow exit passages, J. Phys. Oceanogr., 40, 1965-1987, 2010.

Ducet, N., Traon, P. Y. L., and Reverdin, G.: Global high-resolution mapping of ocean circulation from TOPEX/Poseidon and ERS-1 and -2, J. Geophys. Res., 105, 19,477-19,498, 2000.

Fu, L.-L., Fukumori, I., and Miller, R. N.: Fitting dynamic models to the Geosat sea level observations in the tropical Pacific ocean. Part II: a linear, wind-driven model, J. Phys. Oceanogr., 23, 2162-2181, 1993.

Gentle, J. E.: Random number generation and Monte Carlo methods, Springer, 1998.

Gill, A. E. and Clarke, A. J.: Wind-induced upwelling, coastal currents, and sea-level changes, Deep-Sea Res., $410 \quad 21,325-345,1974$.

Long, R. B. and Thacker, W. C.: Data assimilation into a numerical equatorial ocean model. I. The model and the assimilation algorithm, Dyn. Atmos. Oceans, 13, 379-412, 1989a.

Long, R. B. and Thacker, W. C.: Data assimilation into a numerical equatorial ocean model. II. Assimilation experiments, Dyn. Atmos. Oceans, 13, 413-439, 1989 b.

415 McCreary, J. P.: A linear stratified model of the coastal undercurrent, Philos. Trans. R. Soc. London A, 302 , 385-413, 1981.

Miller, R. N. and Cane, M. A.: A Kalman filter analysis of sea level height in the tropical Pacific, J. Phys. Oceanogr., 19, 773-790, 1989.

Nagura, M. and McPhaden, M. J.: Dynamics of zonal current variations associated with the Indian Ocean dipole,

J. Geophys. Res., 115, C11026, doi:10.1029/2010JC006423., 2010.

Susanto, R. D., Zheng, Q., and Yan, X.-H.: Complex singular value decomposition analysis of equatorial waves in the Pacific observed by TOPEX/Poseidon altimeter, J. Atmos. Oceanic Technol., 15, 764-774, 1998.

Syamsudin, F., Kaneko, A., and Haidvogel, D. B.: Numerical and observational estimates of Indian Ocean Kelvin wave intrusion into Lombok Strait, Geophys. Res. Lett., 31, L24307, 2004.

425 Thacker, W. C. and Long, R. B.: Fitting dynamics to data, J. Geophys. Res., 93, 1227-1240, 1988.

Yu, X. and McPhaden, M. J.: Seasonal variability in the equatorial Pacific, J. Phys. Oceanogr., 29, 925-947, 1999.

Yuan, D. and Liu, H.: Long-wave dynamics of sea level variations during Indian Ocean Dipole events, J. Phys. Oceanogr., 39, 1115-1132, 2009. 
Ocean Sci. Discuss., doi:10.5194/os-2016-1, 2016

Manuscript under review for journal Ocean Sci.

Published: 12 February 2016

(c) Author(s) 2016. CC-BY 3.0 License.

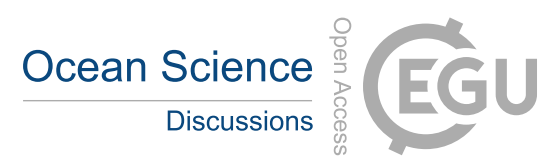

(c) $\underset{\mathrm{BY}}{\mathrm{Br}}$

430 Yuan, D., Rienecker, M. M., and Schopf, P. S.: Long-wave dynamics of the interannual variability in a numerical hindcast of the equatorial Pacific Ocean circulation during the 1990s, J. Geophys. Res., 109, C05019, 2004. 
Ocean Sci. Discuss., doi:10.5194/os-2016-1, 2016

Manuscript under review for journal Ocean Sci.

Published: 12 February 2016

(c) Author(s) 2016. CC-BY 3.0 License.
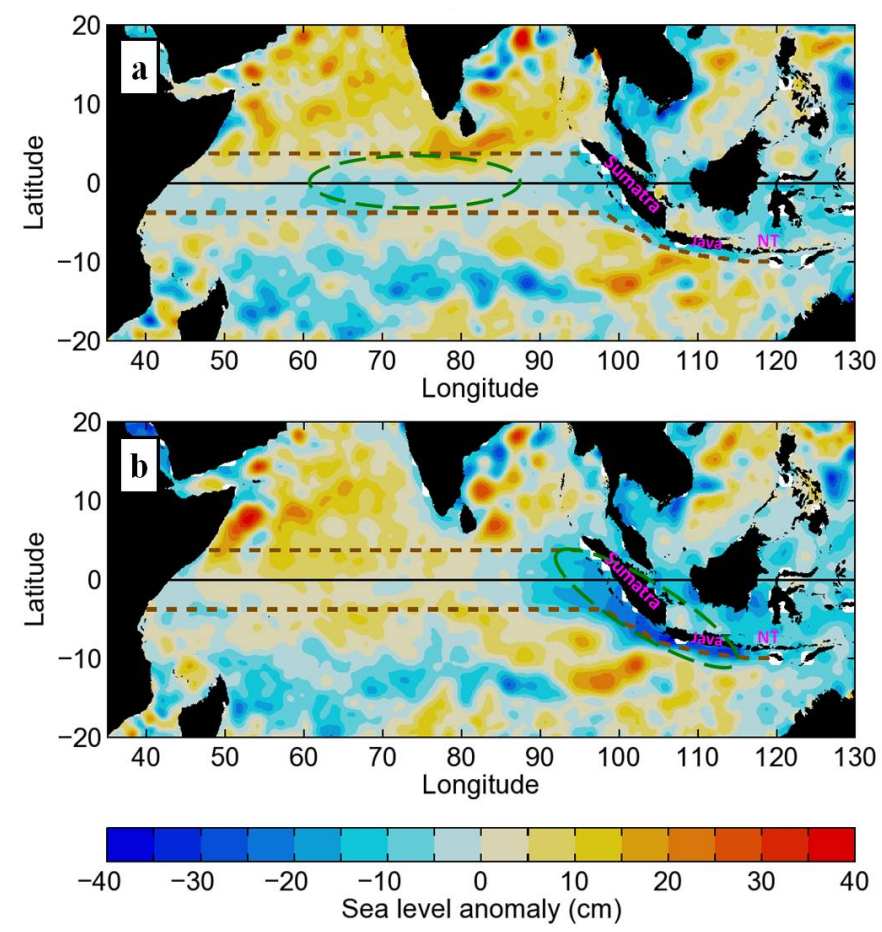

Figure 1. AVISO gridded sea level anomaly (SLA) in the Indian Ocean basin, on (a) June 25, 1997 and (b) July 16, 1997, with upwelling Kelvin waves (depressed SLA) peaking in the central Indian Ocean and along the Sumatra/Java coasts respectively (green dashed ellipses). The brown dashed lines indicate the radii of deformation for 1st baroclinic mode Kelvin waves, with the radius extended along the Indonesian coastal waveguide south of the equator. The locations of Sumatra, Java, and Nusa Tenggara (NT) are also indicated. 
Ocean Sci. Discuss., doi:10.5194/os-2016-1, 2016

Manuscript under review for journal Ocean Sci.

Published: 12 February 2016

(c) Author(s) 2016. CC-BY 3.0 License.
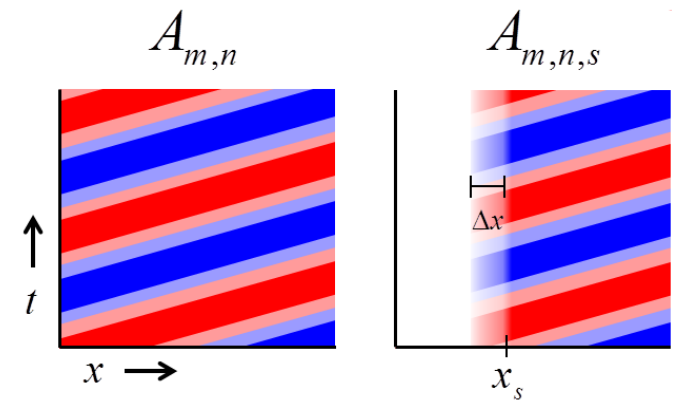

$A_{m, n, s}-A_{m, n, s^{\prime}}$

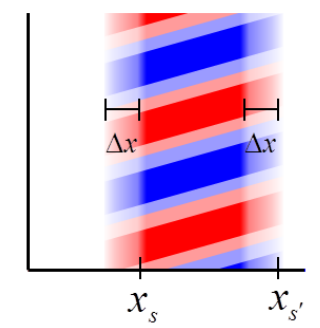

Figure 2. Schematic illustrating the use of tapered basis functions. (a) Profile of a non-tapered two-dimensional basis function $A_{m, n}$ in $x$ and $t$. (b) Profile of a tapered basis function $A_{m, n, s}$, with tapering location $x=x_{s}$ and a tapering window of $\Delta x$. (c) Profile of two superimposed basis functions $A_{m, n, s}-A_{m, n, s^{\prime}}$, with tapering locations of $x=x_{s}$ and $x=x_{s^{\prime}}$ respectively; the superposition of two or more tapered basis functions allows for the forcing and dissipation of waves. 
Ocean Sci. Discuss., doi:10.5194/os-2016-1, 2016

Manuscript under review for journal Ocean Sci.

Published: 12 February 2016

(c) Author(s) 2016. CC-BY 3.0 License.
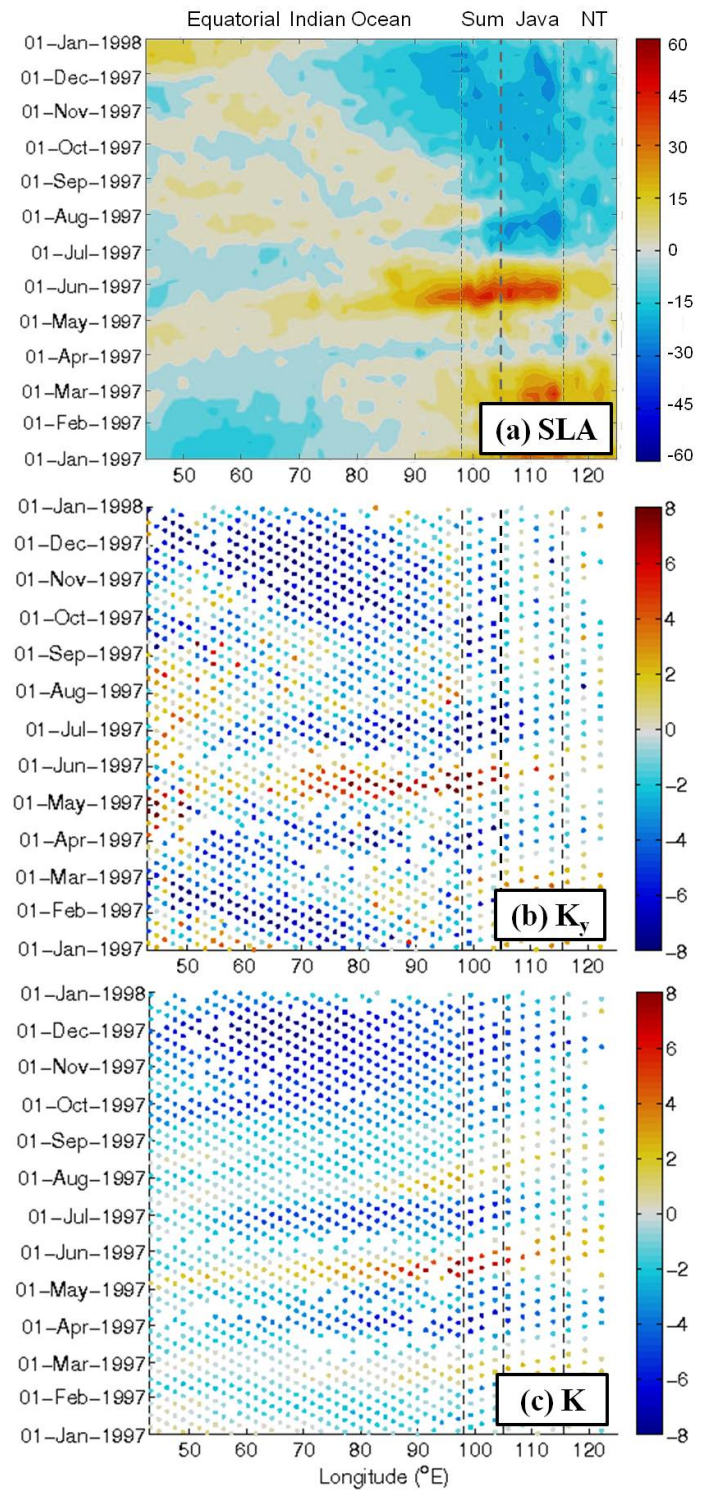

Figure 3. (a) Sea level anomaly (SLA) along the waveguide that crosses the equatorial Indian Ocean and follows the coasts of Sumatra, Java, and Nusa Tenggara, during 1997. The data plotted are from the $1 / 3^{\circ}$ gridded AVISO product, in units of $\mathrm{cm}$. (b) Kelvin wave $y$-projections $K_{y}$ along the waveguide during 1997, derived from the alongtrack SLA data collected by the TOPEX/Poseidon satellite during 1997, in units of $10^{4} \mathrm{~m}^{2}$. (c) The Kelvin wave coefficient $K$ values during 1997, in units of $10^{4} \mathrm{~m}^{2}$. The vertical dashed lines in each plot indicate (from left to right) the locations of the equatorial-coastal transition $\left(98^{\circ} \mathrm{E}\right)$, the Sumatra-Java transition at Sunda Strait $\left(105^{\circ} \mathrm{E}\right)$, and the Java-Nusa Tenggara transition at Lombok Strait $\left(115.65^{\circ} \mathrm{E}\right)$. 
Ocean Sci. Discuss., doi:10.5194/os-2016-1, 2016

Manuscript under review for journal Ocean Sci.

Published: 12 February 2016

(c) Author(s) 2016. CC-BY 3.0 License.
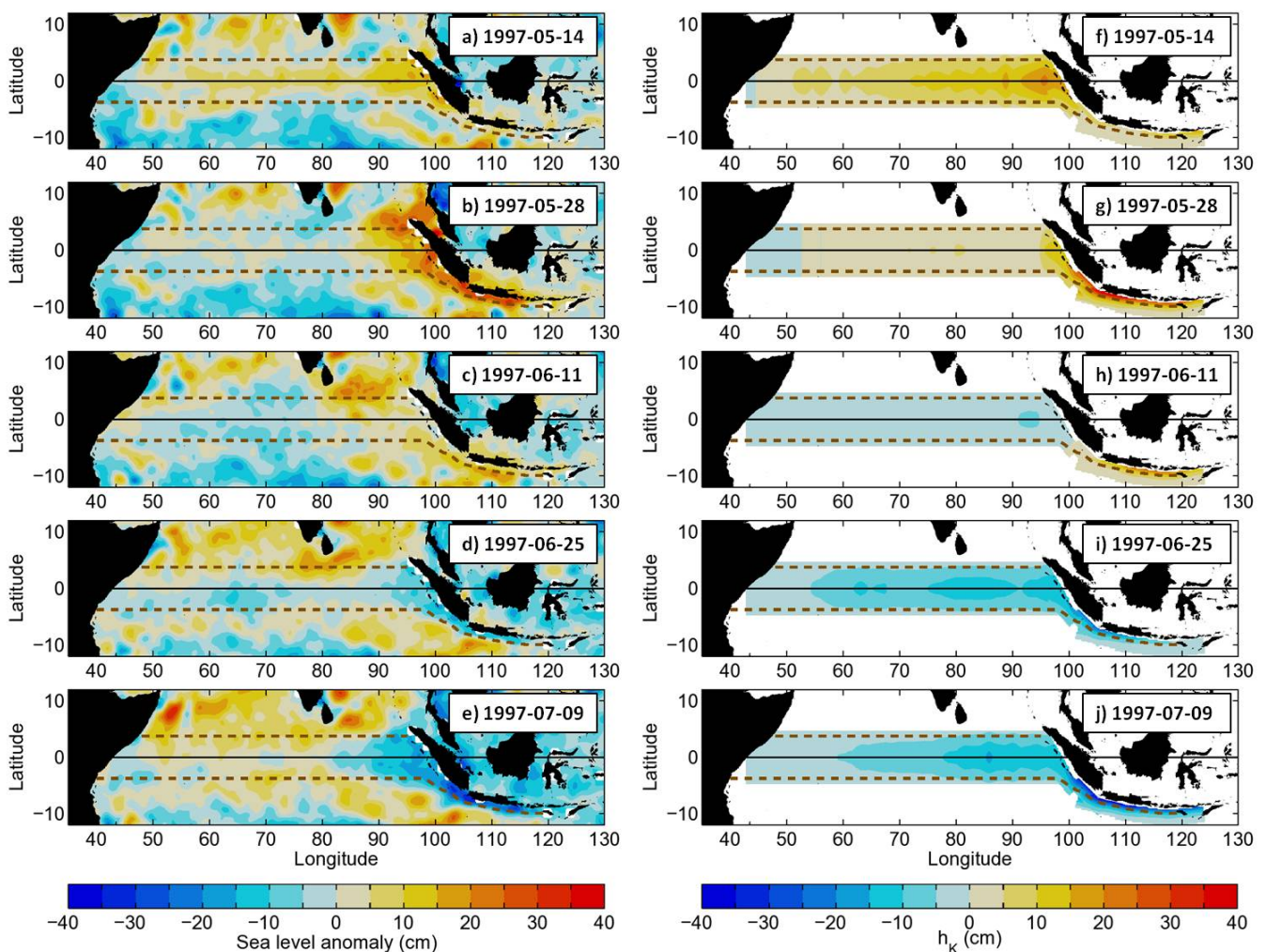

Figure 4. Maps of (a)-(e) SLA and (f)-(j) reconstructed $h_{K}$, the Kelvin-wave associated SLA, for snapshots (dates in the top-right corner of each panel) over a 2-month period in 1997. As in Fig. 1, the brown dashed lines indicate the radii of deformation for 1st baroclinic mode Kelvin waves. 
Ocean Sci. Discuss., doi:10.5194/os-2016-1, 2016

Manuscript under review for journal Ocean Sci.

Published: 12 February 2016

(c) Author(s) 2016. CC-BY 3.0 License.
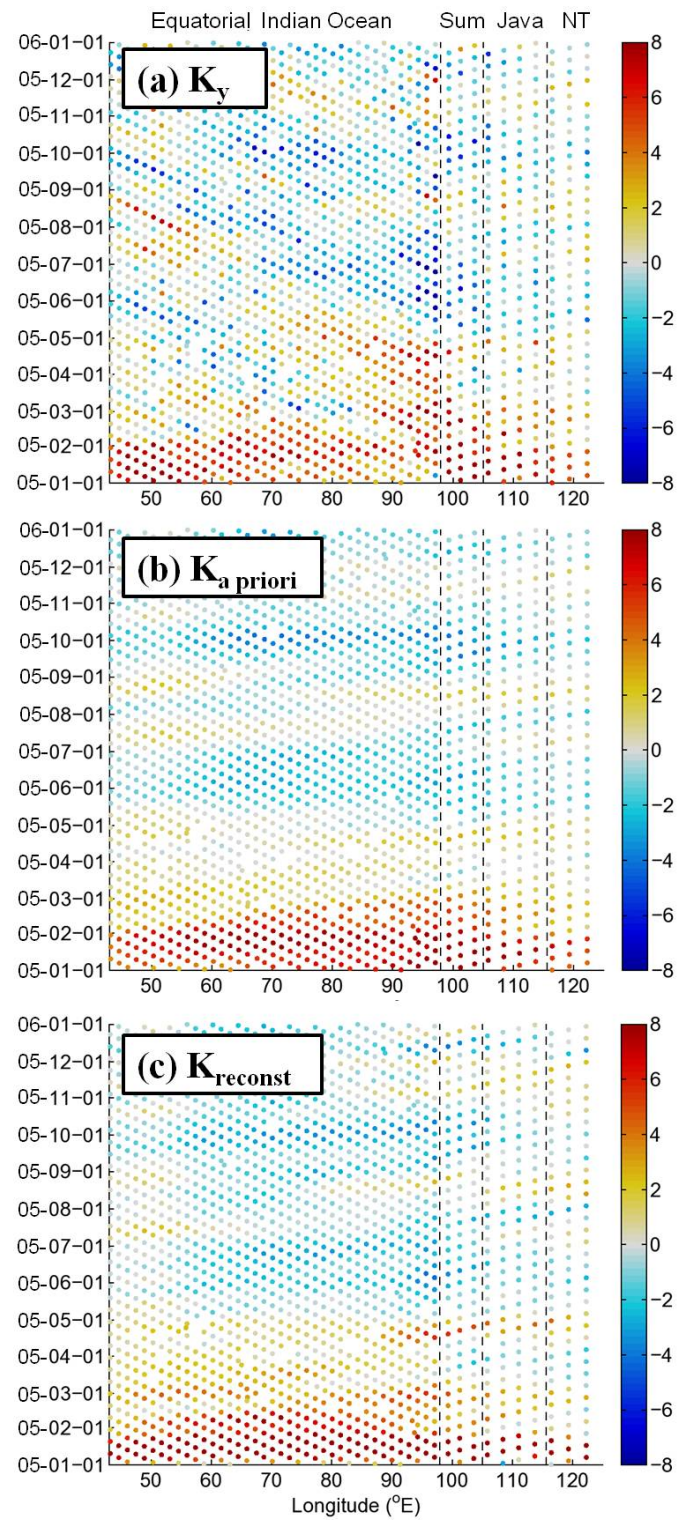

Figure 5. (a) Kelvin wave $y$-projection values $K_{y}$ from year 5 of the Monte Carlo simulation, along the Indian Ocean equatorial-coastal waveguide. (b) Kelvin wave coefficient values $K_{\text {a priori }}$ generated in year 5 of the Monte Carlo simulation. (c) Reconstructed Kelvin wave coefficient values $K_{\text {reconst }}$ for year 5 of the Monte Carlo simulation, obtained using the harmonic projection and least-squares method. All quantities are given in units of $10^{4} \mathrm{~m}^{2}$. 
Ocean Sci. Discuss., doi:10.5194/os-2016-1, 2016

Manuscript under review for journal Ocean Sci.

Published: 12 February 2016
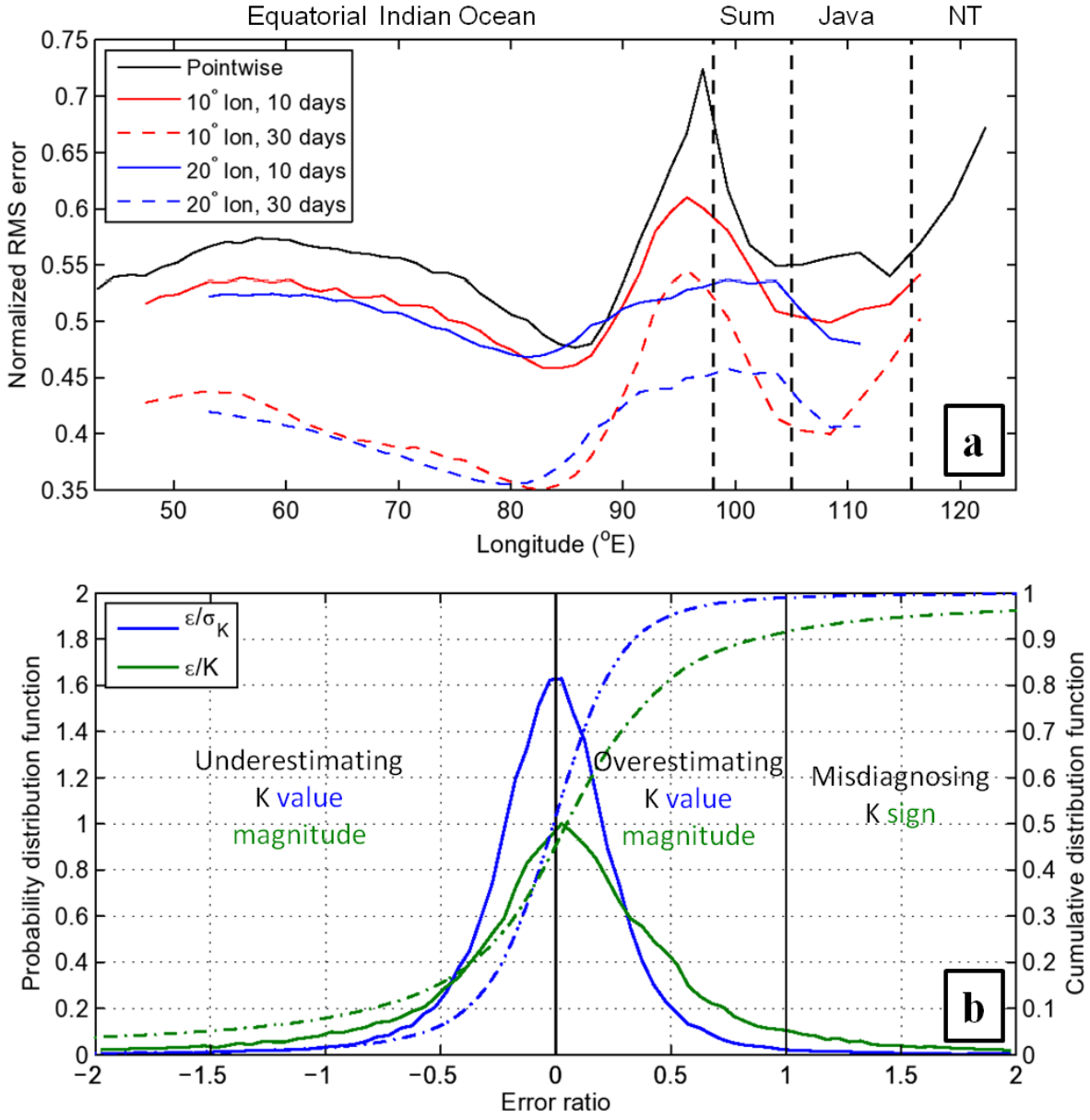

Figure 6. (a) Normalized root-mean-square (RMS) error estimates for $K$ as a function of longitude along the waveguide, based on the 100-year Monte Carlo simulation and computed as $\left\langle\epsilon^{2}\right\rangle^{1 / 2} /\left\langle K^{2}\right\rangle^{1 / 2}$. The different curves show the effect on RMS error of applying spatial and temporal moving average filters to the $a$ priori and reconstructed $K$ values. (b) The probability distribution function (solid lines) and cumulative distribution function (dashed-dotted lines) of normalized error, with a $20^{\circ}$ longitude and 30 day moving average filter applied prior to the error computation. The curves are shown for two different normalizations: (blue) normalized by the standard deviation of $K$ over all longitudes and times $\epsilon / \sigma_{K}$, and (green) normalized by the filtered reconstructed value $K_{\text {reconst }}$ for each longitude and point in time $\epsilon / K_{\text {reconst }}$; in the latter case errors associated withe values of $\left|K_{\text {reconst }}\right|<0.3 \sigma_{K}$ have been excluded. 
Ocean Sci. Discuss., doi:10.5194/os-2016-1, 2016

Manuscript under review for journal Ocean Sci.

Published: 12 February 2016

(C) Author(s) 2016. CC-BY 3.0 License.
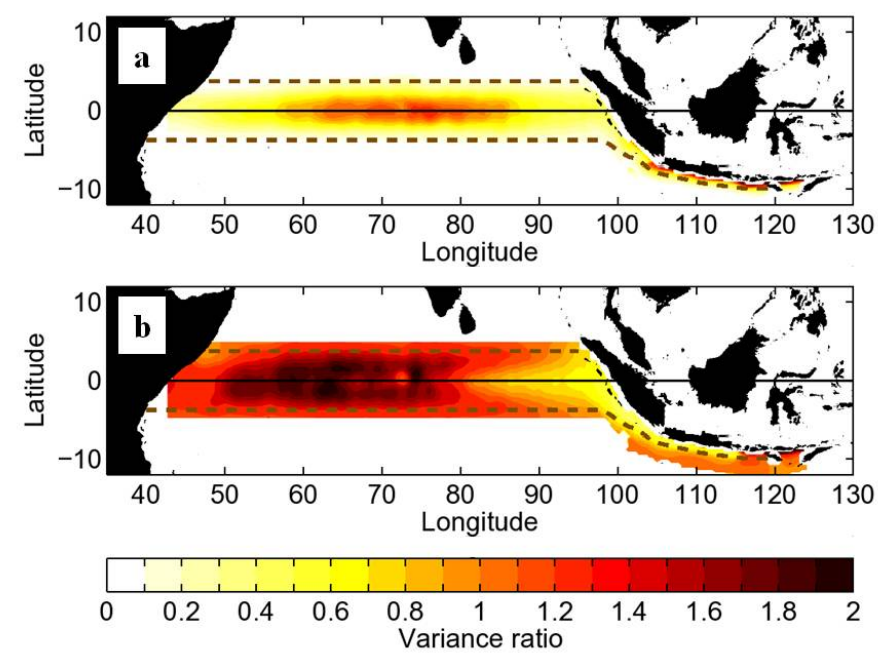

Figure 7. (a) The variance ratio of Kelvin wave-associated SLA to total SLA, $\left\langle h_{K}^{2}\right\rangle /\left\langle h_{\mathrm{SLA}}^{2}\right\rangle$. (b) The variance ratio of the residual to total SLA, $\left\langle h_{\mathrm{res}}^{2}\right\rangle /\left\langle h_{\mathrm{SLA}}^{2}\right\rangle$. The annual and semiannual harmonics have been removed by linear regression.
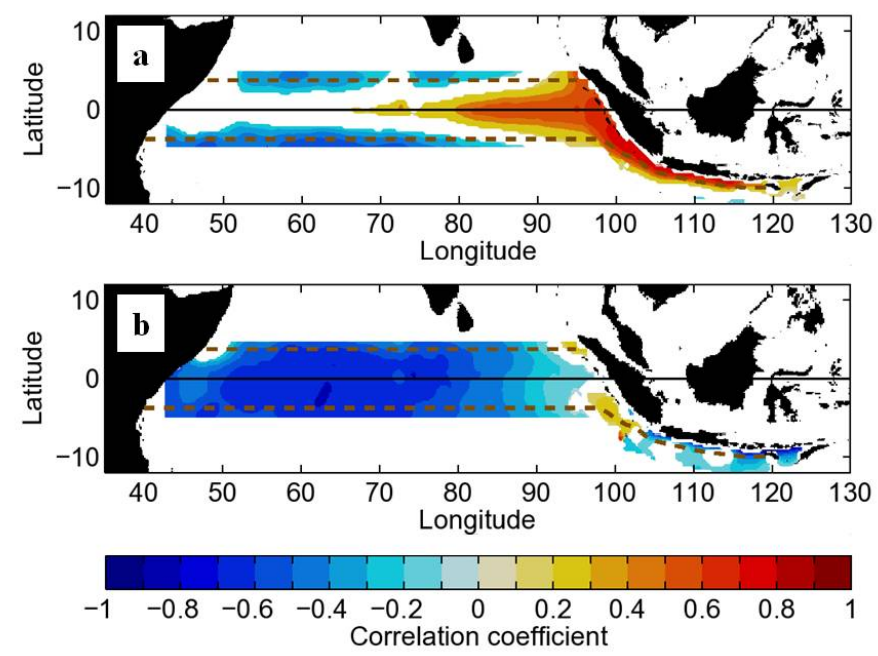

Figure 8. (a) The correlation coefficient of Kelvin wave-associated SLA to total SLA, $\left\langle h_{K} h_{\mathrm{SLA}}\right\rangle /\left[\left\langle h_{K}^{2}\right\rangle\left\langle h_{\mathrm{SLA}}^{2}\right\rangle\right]^{1 / 2}$. (b) The correlation coefficient of Kelvin wave-associated SLA to the residual, $\left\langle h_{K} h_{\mathrm{res}}\right\rangle /\left[\left\langle h_{K}^{2}\right\rangle\left\langle h_{\mathrm{res}}^{2}\right\rangle\right]^{1 / 2}$. The annual and semiannual harmonics have been removed by linear regression. Only correlation coefficients exceeding the 95\% confidence threshold for significance (based on a Student's $t$-distribution) are shaded. 